\title{
Methylprednisolone injection following the surgical extraction of impacted lower third molars: A split-mouth study $^{*}$
}

\author{
Jorge Chaurand-Lara $^{1 \#}$, Jose Alfredo Facio-Umaña ${ }^{2}$ \\ ${ }^{1}$ Department of Oral and Maxillofacial Surgery, National Medical Center "20 de Noviembre" ISSSTE, Ciudad de México, México \\ ${ }^{2}$ Department of Orthodontics, Facultad de Odontología, UnidadTorreón, Universidad Autónoma de Coahuila, Torreón Coahuila, México \\ Email: \#jochala@hotmail.com
}

Received 5 March 2013; revised 26 April 2013; accepted 8 May 2013

Copyright (C) 2013 Jorge Chaurand-Lara, Jose Alfredo Facio-Umaña. This is an open access article distributed under the Creative Commons Attribution License, which permits unrestricted use, distribution, and reproduction in any medium, provided the original work is properly cited.

\begin{abstract}
Purpose: The purpose of the present study was to compare the effect of intramuscular masseter administration of methylprednisolone using a split-mouth design, as a single 20-mg dose, after removal of impacted lower third molars. Patients and Methods: A non-blind, cross-over, comparative, non-randomized, clinical trial was planned. The sample was composed of 32 patients requiring extraction under local anesthesia of two lower bony impacted mandibular third molars. The difficulty of extraction was similar in all cases. The patients received $20 \mathrm{mg}$ of methylprednisolone injected into the masseter muscle via the intrabuccal approach, immediately after suturing of the surgical wound on the right side. The left side received no intramuscular corticoid. Evaluations were made of postoperative pain and swelling. Results: A total of 32 subjects requiring surgical removal of two impacted mandibular third molars under local anesthesia were included in the present study. The patients administered methylprednisolone showed superior results after surgery in terms of pain and facial swelling parameters using self-evaluation, with statistically significant differences versus the control-side $(p<0.05)$. The results obtained show that $20 \mathrm{mg}$ of methylprednisolone injected into the masseter muscle in the immediate postoperative period reduces swelling and pain. Conclusions: Injection of methylprednisolone is an effective therapeutic strategy to reduce swelling and pain after surgical removal of impacted lower third molars. It offers a simple, safe, painless,
\end{abstract}

${ }^{*}$ Conflicts of interest: none declared.

Financial disclosure: none.

${ }^{\#}$ Corresponding author. noninvasive, and cost effective therapeutic option for moderate and severe cases.

Keywords: Methylprednisolone; Third Molar Surgery; Split-Mouth Study

\section{INTRODUCTION}

The extraction of impacted lower third molars is the most common operation in oral surgery and usually produces pain, trismus and facial swelling in the postoperative period [1], because the region of surgery is mostly composed of loose connective tissue that contains blood and lymph vessels, a series of functional and structural alterations is expected after extraction, mostly expressed as pain, swelling, and trismus [2].

To reduce postoperative complications, therefore, seems a logical goal, particularly if healing is not compromised, and the use of corticosteroids has gained wide acceptance [3]. These agents act by inhibiting the body's inflammatory response to injury through various mechanisms, with a reduction of fluid transudation and therefore oedema [4]. Over several decades many studies have reported the effectiveness of corticosteroids given before or just after removal of third molars in improving recovery [5-12]. The method of use, however, has varied, and the most effective regimen has yet to be defined. Reports of corticosteroids given in the region adjacent to the site of operation, showed encouraging results compared with controls [13-15]. The technique is convenient for the surgeon, as the injection is given in close proximity to the operative field, and for the patient, as the injection is given into an anaesthetised area.

The glucocorticoids most widely used in oral surgery are dexamethasone (p.o.), dexamethasonesodium phos- 
phate (i.v. and i.m.), dexamethasone acetate (i.m.), methylprednisolone (p.o.), and methylprednisolone acetate and methylprednisolone sodium succinate (i.v. and i.m.) [1].

The aim of the present clinical study was to evaluate the effect of a single $20-\mathrm{mg}$ dose of methylprednisolone acetate into the masseter muscle via the intrabuccal approach following the surgical extraction of impacted lower third molars under local anaesthesiausing a split-mouth design. A study hypothesis was formed stating that "methylprednisolone acetate injection provides a significant improvement of VAS measures (pain and swelling) after third molar surgery comparable to non-injection side".

\section{MATERIALS AND METHODS}

32 healthy patients (American Society of Anesthesiologists classifications I or II) from both genders, ranging in age from 16 to 50 years, with no periodontal disease and with indication for removal of two mandibular third molars, were included in this study, after signing a detailed informed consent. In addition, to standardize the sample, there had to be similar patterns of teeth and root formation, position, and degree of impaction between right and left sides of the mouth. Also orthopantomography showed positioning of the teeth to be symmetrical.

Exclusion criteria consisted of tobacco use, patients with orthodontic bans on the second molars, pregnant or breast-feeding women, contraceptive users, patients with chronic system disorders, patients with a history of allergies or adverse effects associated with antibiotics and analgesics used in the study, and individuals who had used antibiotics over the previous 3 months. On the day of surgery, presence of any pre-existing acute inflammatory or infectious condition was also grounds for exclusion. When a procedure required more than 1.5 hours or there was a significant difference between the right side and left side procedure, the patient was removed from the study sample. The patients were blinded to the use of corticosteroids. The local institutional review board reviewed and approved the research study.

Each patient was submitted to 2 surgical procedures in 1 clinical session for removal of 2 molars. The Student's t-test for related samples was used to compare qualitative means. The SPSS version 11.0 statistical package was used throughout.

\section{OPERATIVE TECHNIQUE}

The same surgeon operated on all patients using a standard technique. Anaesthesia was by a standard inferior alveolar nerve block and long buccal nerve block using a solution of $2 \%$ lidocaine hydrochloride with epinephrine 1:100,000. Surgical access was by a standard triangular mucoperiosteal flap. Bone was then removed around the tooth with a round bur on a straight hand piece under continuous irrigation with dilute saline solution. The crown or roots were sectioned when necessary. After complete extraction of the tooth (or its components), the socket was inspected, irrigated copiously, and the flap was sutured back. Following removal of the third molar in the corticoid side (right), $20 \mathrm{mg}$ of methyl prednisolone acetate was injected into the masseter muscle via the intrabuccal approach. The same procedure was made on control group (left side) but received no corticoid. A small gauze pack was then applied to the site and the usual postoperative instructions given to the patient. Apart from treatment, all patients in the study routinely received amoxicillin (oral $500 \mathrm{mg}$ every 8 hours) for 5 days after surgery and $500 \mathrm{mg}$ acetaminophen were administered orally every 6 hours.

One day after surgery the patients rated pain on a 10 $\mathrm{cm}$ visual analog scale, the extreme scores being "no pain" and "worst pain imaginable". Swelling was assessed subjectively by asking participants to look into a mirror and mark on a separate $10-\mathrm{cm}$ visual analogue scale the level of facial swelling they felt was present.

\section{RESULTS}

The mean patient age (14 men and 18 women) was 23.31 $+/-10.03$ years (range 14 to 50 ). No statistically significant differences were found in the demographic and clinical characteristics among the study groups. No data were missing, and all patients included in the present study attended all study visits. At follow-up, no cases of alveolar osteitis or wound infection were reported. No side effects of the drugs used in the trial were mentioned or noted.

In 24 patients, pain (using the visual analog scale) were less severe on postoperative day 1 comparing to non-steroid side (control group). One patient remained without pain difference between groups, 3 patients reported no pain on either side and 4 patients had more pain on the right side (Figure 1).

In 25 patients, swelling (using the VAS) were less severe on postoperative day 1 comparing to the control group, 1 patient remained without change between right and left side, 6 patients had more severe edema on the right side (Figures 2 and $\mathbf{3}$ ).

The steroid side showed a statistically significant difference in the magnitude of swelling and pain at all intervals $(\mathrm{p}<0.05)$ (Table 1$)$.

The VAS scores were divided according to magnitude; 1 - 3: moderate, 4 - 6: mild, severe: 7 - 10 (Table 2).

\section{DISCUSSION}

The split-mouth design is a popular design in oral health research. In the most common split-mouth study, each of 


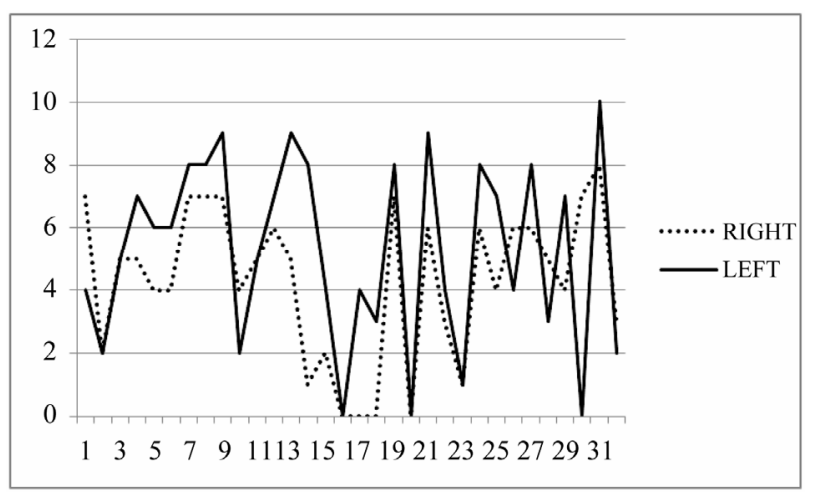

Figure 1. Pain VAS measurements between left and right side.

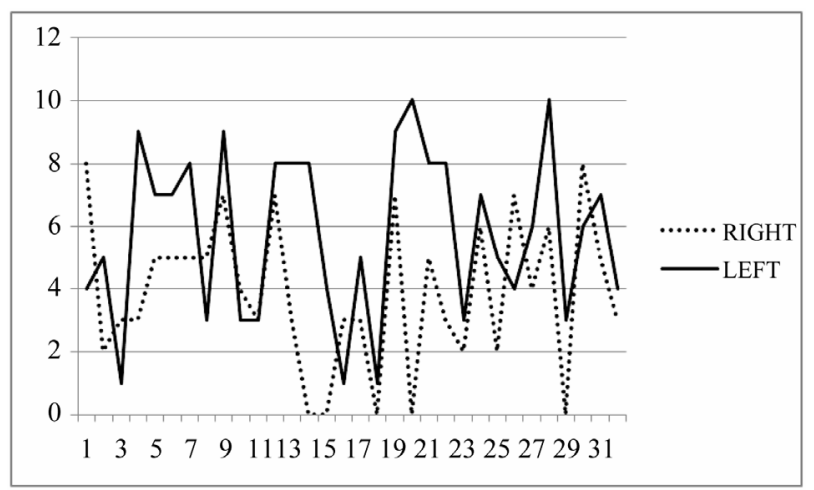

Figure 2. Swelling VAS measurements between left and right side.

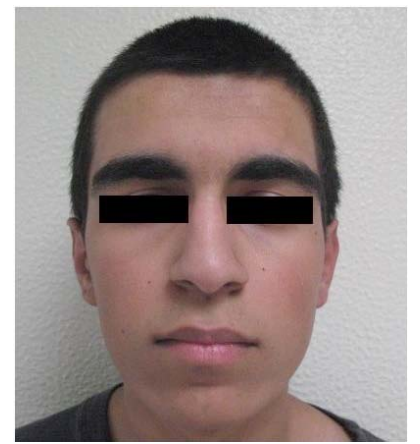

(a)

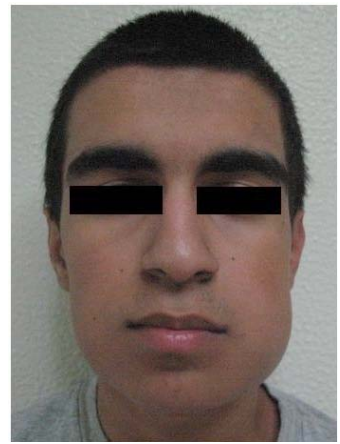

(b)
Figure 3. (a) Preoperative clinical view; (b) Postoperative clinical view.

Table 1. Mean values and standard deviations between left and right side.

\begin{tabular}{cccccc}
\hline & \multicolumn{2}{c}{ Swelling } & \multicolumn{2}{c}{ Pain } & \multirow{2}{*}{ p value } \\
\cline { 2 - 5 } & Mean & SD & Mean & SD & \\
\hline Right & 3.87 & 2.41 & 4.28 & 2.43 & $<0.001$ \\
Left & 5.75 & 2.68 & 5.25 & 2.97 & $<0.001$ \\
\hline
\end{tabular}

Abbreviations: SD: standard deviation.

two treatments are randomly assigned to either the right or left halves of the dentition. The attractiveness of the
Table 2. Pain and swelling scores according to VAS on posoperative day 1 .

\begin{tabular}{|c|c|c|c|c|c|c|}
\hline \multirow[t]{2}{*}{$\mathrm{N}(32)$} & \multicolumn{2}{|c|}{ Pain (VAS) } & \multirow[t]{2}{*}{$\mathrm{N}(32)$} & \multicolumn{2}{|c|}{ Swelling (VAS) } & \multirow[t]{2}{*}{$\mathrm{p}$ value } \\
\hline & RS & LS & & RS & LS & \\
\hline 24 & $1-3$ & $7-10$ & 25 & $1-3$ & $7-10$ & $<0.001$ \\
\hline 1 & $7-10$ & $7-10$ & 1 & $1-3$ & $1-3$ & $<0.001$ \\
\hline 3 & $1-3$ & $1-3$ & 6 & $7-10$ & $1-3$ & $<0.001$ \\
\hline 4 & $7-10$ & $1-3$ & & & & $<0.001$ \\
\hline
\end{tabular}

Abbreviations: VAS: visual analogue scale, RS: right side (corticoid side), LS: left side.

design is that it removes a lot of inter-individual variability from the estimates of the treatment effect [16], also split-mouth designs are efficient because they require smaller sample sizes [17]. The value of including patient-reported outcomes in audit and clinical research is now well recognized. It has been found that surgeonrated scores or objective testing are significantly different from the patients' perspective $[18,19]$.

Standardization of the medication used was important to reduce drug-related bias. However, the effect on postoperative morbidity, and the duration of the effect of the corticosteroids, varied, mainly as a result of lack of consensus about the optimal route, dose, timing, and duration of treatment in addition to differences in methods used to evaluate clinical variables. The intramuscular masseteric route, however, has been reported on only isolated occasions and was not mentioned even in most recent reviews [5-8].

Methylprednisolone has been used at a constant dosage in most studies [7]. Esen et al. (1999) [10] reported that a single intravenous dose of $125 \mathrm{mg}$ of methylprednisolone reduces the swelling, pain and trismus associated with third molar surgery. This coincides with the present observations, although involving a different dose and route of administration. Huffman (1977) [20] compared the intravenous administration of $40 \mathrm{mg}$ and 125 $\mathrm{mg}$ of methylprednisolone in extractions of impacted lower third molars. Although the increase in dose further reduced swelling, the difference was not statistically significant. Ustün et al. (2003) [11] evaluated the effects of $1.5 \mathrm{mg} / \mathrm{kg}$ and $3 \mathrm{mg}$ of methylprednisolone via the intravenous route in terms of the postoperative complications (pain, swelling and trismus) recorded after third molar surgery. No significant differences were observed between the two groups, and the authors concluded that administration of a higher methylprednisolone dose affords no clinical benefit.

The intramuscular route affords good plasma drug concentrations and prolonged anti-inflammatory action with a single pre- or postoperative dose [8]. MicóLlorens et al. (2006) [14] injected $40 \mathrm{mg}$ of methylprednisolone into the gluteal zone following the extraction of 
impacted third molars, and reported good results 2 days after the operation in terms of swelling, pain and trismus, but after 7 days the differences were no longer significant. Messer \& Keller (1975) [21] injected dexamethasone into the masseter muscle and reported a decrease in pain, swelling and trismus, similarly to the present results with methylprednisolone. The administration of corticosteroids effectively reduces trismus, pain and facial swelling, the selected corticosteroid should have scant mineralocorticoid effects and great biological activity; methylprednisolone meets these requirements, since it has no mineralocorticoid activity, the half-life is approximately 18 - 36 hours and the drug is 5-fold more potent than hydrocortisone [1].

According to recent reports [1,3,19] of corticosteroids given in the region adjacent to the site of operation, the first day after surgery showed the higher values in terms of pain and swelling, that's why we decided to conduct VAS measures only on this day so we can get a specific effect of the methylprednisolone comparing with controlside for the potencially "worst" postoperative day for the patients.

The main results of the present study were that intramuscular masseteric injections of $20 \mathrm{mg}$ methylprednisolone just after surgery resulted in significant improvement in swelling and pain measures on the first postoperative day compared with control-side. Finally, the effects in the methylprednisolone side were comparable for all parameters and at all intervals. The response rate to the study was high, indicating the high feasibility of using patient-centered outcome measures in oral surgery. It could also indicate the clinical validity of the VAS used in the present study, reflected by its ability to discriminate among different groups of patients and to correlate very well with the objectively measured swelling and pain. There are limitations to this study, VAS were performed only on the first postoperative day and the patients were not randomized into the respective test groups, a potencial for selection bias may be introduced.

Overall, the results of the present study suggest the advantage of methylprednisolone injected adjacent to the site of operation as an effective alternative to systemically applied steroids. The technique is quite simple, less invasive, painless (given in an anesthetized region), and convenient for the surgeon and patient and offers a lowcost solution for the typical patient discomfort associated with the surgical extraction of impacted lower third molars. Injection after surgery offers the advantage of concentrating the drug near the surgical area with less systemic absorption. This timing also allows the surgeon to accurately assess the need for the steroid injection according to the postoperatively recorded surgical difficulty and duration of intervention [19]. Extracting both lower third molars in the same operation is commonly done, the surgeon can also assess the need to inject both sides for a total dose of $40 \mathrm{mg}$.

The absolute contraindications to corticosteroid use include patients with tuberculosis, active viral or fungal infections, active acne vulgaris, primary glaucoma, a history of acute psychosis or psychopathic tendencies and allergies [8]. Many studies have shown that pain decrease with steroids, but a clear pathway for this effect has not been explained. Some authors suggest that the swelling made the tissue tense and caused tension pain that was reduced when steroids decreased the facial swelling [22].

Additional studies are required to confirm the effectiveness of locally administered corticosteroids in third molar surgery compared with other commonly used routes. Larger randomized patient samples are also needed to evaluatethe potential adverse effects of locally applied corticosteroids on wound healing after surgery.

\section{ACKNOWLEDGEMENTS}

We would to thank Dr. Laura Pacheco, Dr. Nancy Bertado Ramirez and Dr. Javier Moran Martinez for their technical assistance in the preparation of this article.

\section{REFERENCES}

[1] Vegas-Bustamante, E., Mico-Llorens, J., Gargallo-Albiol, J., Satorres-Nieto, M., Berini-Aytes, L. and Gay-Escoda, C. (2008) Efficacy of methylprednisolone injected into the masseter muscle following the surgical extraction of impacted lower third molars. International Journal of Oral and Maxillofacial Surgery, 37, 260-263. doi:10.1016/i.ijom.2007.07.018

[2] Laureano-Filho, J.R., Maurette, P.E., Allais, M., et al. (2008) Clinical comparative study of the effectiveness of two dosages of dexamethasone to control postoperative swelling, trismus and pain after the surgical extraction of mandibular impacted third molars. Medicina Oral Patologia Oral Cirugia Bucal, 13, 129.

[3] Majid, O.W. and Mahmood, W.K. (2011) Effect of submucosal and intramuscular dexamethasone on postoperative sequelae after third molar surgery: Comparative study. British Journal of Oral and Maxillofacial Surgery, 49, 647-652. doi:10.1016/j.bjoms.2010.09.021

[4] Milles, M. and Desjardins, P. (1993) Reduction of postoperative facial swelling by low-dose methylprednisolone: An experimental study. Journal of Oral and Maxillofacial Surgery, 51, 987-991. doi:10.1016/S0278-2391(10)80041-2

[5] Skjelbred, P. and Lokken, P. (1982) Post-operative pain and inflammatory reaction reduced by injection of a corticosteroid. A controlled trial in bilateral oral surgery. European Journal of Clinical Pharmacology, 21, 391396. doi:10.1007/BF00542325

[6] Pedersen, A. (1985) Decadron phosphate in the relief of complaints after third molar surgery. A double-blind, controlled trial with bilateral oral surgery. International 
Journal of Oral Surgery, 14, 235-240. doi:10.1016/S0300-9785(85)80034-X

[7] Beirne, O.R. and Hollander, B. (1986) The effect of methylprednisolone on pain, trismus, and swelling after removal of third molars. Oral Surgery, Oral Medicine, Oral Pathology, 61, 134-138. doi:10.1016/0030-4220(86)90173-8

[8] Montgomery, M.T., Hogg, J.P., Roberts, D.L. and Redding, S. (1990) The use of glucocorticosteroidsto lessen the inflammatory sequelae following third molar surgery. Journal of Oral and Maxillofacial Surgery, 48, 179-187. doi:10.1016/S0278-2391(10)80207-1

[9] Neupert III, E.A., Lee, J.W., Philput, C.B. and Gordon, J.R. (1992) Evaluation of dexamethasonefor reduction of postsurgical sequelae of third molar removal. Journal of Oral and Maxillofacial Surgery, 50, 1177-1182. doi:10.1016/0278-2391(92)90149-T

[10] Esen, E., Tasar, F. and Akhan, O. (1999) Determination of the anti-inflammatory effects of methylprednisolone on the sequelae of third molar surgery. Journal of Oral and Maxillofacial Surgery, 57, 1201-1206. doi:10.1016/S0278-2391(99)90486-X

[11] Ustün, Y., Erdogan, Ö., Esen, E. and Karsli, E.D. (2003) Comparison of the effects of 2 doses of methylprednisolone on pain, swelling, and trismus after third molar surgery. Oral Surgery, Oral Medicine, Oral Pathology, Oral Radiology, and Endodontics, 96, 535-539. doi:10.1016/S1079-2104(03)00464-5

[12] Tiwana, P.S., Foy, S.P., Shugars, D.A., Marciani, R.D., Conrad, S.M., Phillips, C., et al. (2005) The impact of intravenous corticosteroids with third molar surgery in patients at high risk for delayed health-related quality of life and clinical recovery. Journal of Oral and Maxillofacial Surgery, 63, 55-62. doi:10.1016/j.joms.2004.01.029

[13] Graziani, F., D'Aiuto, F., Arduino, P.G., Tonelli, M. and Gabriele, M. (2006) Perioperative dexamethasone reduces post-surgical sequelae of wisdom tooth removal. A splitmouth randomized double-masked clinical trial. International Journal of Oral and Maxillofacial Surgery, 35, 241-246. doi:10.1016/j.ijom.2005.07.010

[14] Mico-Llorens, J.M., Satorres-Nieto, M., Gargallo-Albiol, A., Arnabat-Dominguez, J., Berini-Aytes, L. and Gay-
Escoda, C. (2006) Efficacy of methylprednisolone in controlling complications after impacted lower third molar surgical extraction. European Journal of Clinical Pharmacology, 62, 693-698. doi:10.1007/s00228-006-0164-5

[15] Grossi, G.B., Maiorana, C., Garramone, R.A., Borgonovo, A., Beretta, M., Farronato, D., et al. (2007) Effect of submucosal injection of dexamethasone on postoperative discomfort after third molar surgery: A prospective study. Journal of Oral and Maxillofacial Surgery, 65, 22182226. doi:10.1016/j.joms.2006.11.036

[16] Lesaffre, E., Philstrom, B., Needleman, I. and Worthington, H. (2009) The design and analysis of split-mouth studies: What statisticians and clinicians should know. Statistics in Medicine, 28, 3470-3482. doi:10.1002/sim.3634

[17] Pandis, N. (2012) Sample calculation for split-mouth designs. American Journal of Orthodontics and Dentofacial Orthopedics, 141, 818-819. doi:10.1016/j.ajodo.2012.03.015

[18] Monaco, G., Tavernese, L., Agostini, R., et al. (2009) Evaluation of antibiotic prophylaxis in reducing postoperative infection after mandibular third molar extraction in young patients. Journal of Oral and Maxillofacial Surgery, 67, 1467. doi:10.1016/j.joms.2008.12.066

[19] Majid, O.W. (2011) Submucosal dexamethasone injection improves quality of life measures after third molar surgery: A comparative study. Journal of Oral and Maxillofacial Surgery, 69, 2289-2297. doi:10.1016/j.joms.2011.01.037

[20] Huffman, G. (1977) Use of methylprednisolone sodium succinate to reduce postoperative edema after removal of impacted third molars. Journal of Oral Surgery, 35, 198199.

[21] Messer, E. and Keller, J. (1975) The use of intraoral dexamethasone after extraction of mandibular third molars. Oral Surgery, Oral Medicine, Oral Pathology, 40, 594598. doi:10.1016/0030-4220(75)90369-2

[22] Boworn, K., Pariya, K., Kiatanant, B. and Natthamet, W. (2012) Effect of single dose preoperative intramuscular dexamethasone injection on lower impacted third molar surgery. International Journal of Oral and Maxillofacial Surgery, 41, 376-379. 